\title{
Quantification of the association of ventilation rates with sick building syndrome symptoms
}

\author{
William J, Fisk ${ }^{*}$, Anna G. Mirer and Mark J. Mendell \\ Indoor Environment Department, Lawrence Berkeley National Laboratory, Berkeley, CA \\ ${ }^{*}$ Corresponding email: wjfisk@lbl.gov
}

\begin{abstract}
SUMMARY
Data from published studies were combined and analyzed to develop best-fit equations and curves quantifying the change in sick building syndrome (SBS) symptom prevalence with ventilation rate. For each study, slopes were calculated, representing the fractional change in SBS symptom prevalence per unit change in ventilation rate per person. Values of ventilation rate, associated with each value of slope, were also calculated. Linear regression equations were fit to the resulting data points, after weighting by study size. Integration of the slopeventilation rate equations yielded curves of relative SBS symptom prevalence versus ventilation rate. Based on these analyses, relative SBS symptom prevalence increases approximately $23 \%$ (12\% to $32 \%$ ) as the ventilation rate drops from 10 to $5 \mathrm{~L} / \mathrm{s}$-person and relative prevalence decreases approximately $29 \%$ (15\% to $42 \%)$ as ventilation rate increases from 10 to $25 \mathrm{~L} / \mathrm{s}$-person.
\end{abstract}

\section{KEYWORDS}

Association, Health, Quantification, Sick building syndrome, Symptoms, Ventilation rate

\section{INTRODUCTION}

Numerous experimental and cross-sectional studies have investigated the associations of the prevalence rates of sick building syndrome (SBS) symptoms with office building ventilation rates. Studies have typically used the odds ratio or relative risk to indicate strength of the association of SBS symptom prevalence with ventilation rates and have generally controlled via the study design or analysis method for several potential confounding factors. Although ventilation rates, SBS symptom types, and findings have varied among studies, most studies have found that occupants of office buildings with lower rates of ventilation (outdoor air supply) per person have statistically significantly higher prevalence rates of SBS symptoms (Seppanen et al. 1999; Wargocki et al. 2002). For this paper, we have analyzed data to develop best-fit equations quantifying the change in relative SBS symptom prevalence with ventilation rate. A prior similar analysis quantified the relationship of relative work performance with ventilation rate (Seppanen et al. 2006). By quantifying these and other relationships between IAQ factors and health or performance outcomes, we seek to provide tools that better inform decisions about building design or operation.

\section{METHODS}

\section{Overall approach}

The overall approach employed in this study is indicated by the following steps:

- establishment of criteria for input data;

- searching for and obtaining data (i.e., documents with data) satisfying the criteria; 
- extraction and analyses of applicable data from each accepted source to provide slopes (changes in SBS symptom prevalence divided by the average prevalence divided by changes in ventilation rate) and corresponding central values of ventilation rate;

- statistical analyses to fit equations to the resulting data;

- integration of the best-fit equations to provide equations for the change in relative SBS symptom prevalence versus ventilation rate.

\section{Selection of input data}

This analysis started with data provided in technical papers or reports from numerous specific research studies. Useable papers must have provided the odds ratio or relative risk for the change in symptom prevalence or intensity with change in ventilation rate, the fractional change in symptom prevalence or intensity with change in ventilation rate, or symptom prevalence or intensity data at different ventilation rates. The magnitude of the change in ventilation rate per person must be provided or quantifiable from the study data. The number of study subjects must be provided to enable weighting of each study in a statistical analysis. We accepted data from articles in refereed archival journals, papers published in conference proceedings, and reports from research institutions.

Ideally, all of the data used in our analyses would come from studies with identical methodologies and SBS symptom definitions. In practice, however, the number of studies providing suitable data was limited and these studies have used a range of research methods and SBS symptom definitions. Consequently, in order to have enough data for analyses, we accepted the results from a variety of study types and with a range of SBS outcome definitions. We accepted data from both cross sectional and experimental studies as long as the study design or analyses controlled for potential confounding by gender, HVAC type, and temperature, and the subjects were "blinded" (not informed) with respect to ventilation rates. We required that data come from studies of office buildings with office workers as the subjects and that symptom data be from questionnaires completed by the subjects. Within the subjective data on SBS symptoms, there remained a wide range in data types including various symptom types (e.g., eye irritation, skin irritation, headache, difficulty breathing, etc.). Although the ventilation-symptom relationship might vary among symptom types, given our objective of developing an estimate of the average quantitative ventilation-symptom relationship, and given the paucity of data, all of these types of symptoms were accepted. However, we excluded environmental perception outcomes (e.g., stuffy air) which were sometimes treated as "symptoms" by authors of papers. We accepted data from analyses with symptoms grouped into categories, e.g., mucous membrane symptoms. Symptom prevalence data were accepted regardless of the recall period (e.g., past week, past month); however, for some analyses data from questionnaires that asked about current or current-day symptom intensity, as opposed to symptom prevalence, were excluded.

The methods used to measure ventilation rates varied among studies. We accepted data based on the following measurement methods:

A. use of flow hoods or similar methods to measure supply air flow rates to rooms or zones of buildings with HVAC systems that supply $100 \%$ outdoor air;

B. measurement of carbon dioxide concentrations in occupied spaces and use of mass balance equations to calculate ventilation rates;

C. use of tracer gas decay methods or other tracer methods; and

D. use of anemometers to measure air flow rates through the outdoor-air intake sections of HVAC systems with $100 \%$ outdoor air supply. 


\section{Data analysis procedures}

There were three basic steps to the data analysis process. First, the data in the original papers were processed to determine normalized slopes (fractional changes in SBS symptom prevalence divided by changes in ventilation rate) and the corresponding central values for the ventilation rate interval were calculated. Second, a statistical program was used to fit equations to the resulting pairs of numbers (slopes and ventilation rates). Third, these equations were integrated and used to calculate equations of relative SBS symptom prevalence versus ventilation rate. The protocols used in each step are described below.

The methods employed to calculate slopes, i.e., change in SBS symptom prevalence divided by associated change in ventilation rate, varied with the form of the original data. However, the goal was a normalized slope defined as follows

$$
S=\left(\frac{P_{H}-P_{L}}{P_{H}}\right) /\left(V_{H}-V_{L}\right)
$$

where $S$ is the slope, $P$ is a SBS symptom prevalence, $\mathrm{V}$ is a ventilation rate per person, and subscripts $H$ and $L$ refer to the high ventilation rate and low ventilation rate conditions, respectively. As defined above, the slope is positive if the SBS symptom prevalence increases with an increase in ventilation rates, although the opposite is usually true in practice.

The slope at the midpoint ventilation rate between $P_{H}$ and $P_{L}$ is

$$
S_{\text {mid }}=\left(\frac{P_{H}-P_{L}}{P_{m i d}}\right) /\left(V_{H}-V_{L}\right)
$$

where the subscript "mid” refers to a value at the midpoint ventilation rate.

The midpoint ventilation rate $V_{\text {mid }}$ is

$$
V_{\text {mid }}=\left(V_{H}+V_{L}\right) / 2
$$

The point estimate of $S_{\text {mid }}$ at the midpoint of ventilation rates in each study is calculated as shown in equation 4 . The equation is based on the fact that, assuming a locally approximately linear relationship between SBS symptom prevalence $P$ and ventilation rate from $V_{L}$ to $V_{H}$, the prevalence at the midpoint $\left(P_{\text {mid }}\right)$ equals $P_{H} \cdot\left[1-0.5 \cdot\left(V_{H}-V_{L}\right) \cdot S\right]$.

$$
S_{\text {mid }}=\frac{S}{1-\left[0.5 \cdot S \cdot\left(V_{H}-V_{L}\right)\right]}
$$

Some experimental studies provided symptom prevalence data for the high and low ventilation rate conditions. In these cases, equation 1 was used directly to calculate S. Some studies provided values of relative risk for SBS symptoms. The relative risk $R R$ is related to symptom prevalence as follows 


$$
R R=P_{L} / P_{H}
$$

From algebraic manipulations

$$
1-R R=\left(P_{H}-P_{L}\right) / P_{H}
$$

thus,

$$
S=(1-R R) /\left(V_{H}-V_{L}\right)
$$

Some studies provided adjusted odds ratios for SBS symptoms. The odds ratio $(O R)$ is defined as follows

$$
O R=\frac{P_{L}}{1-P_{L}} / \frac{P_{H}}{1-P_{H}}
$$

For symptom prevalence rates less than approximately $15 \%$, the $O R$ is numerically very close to the $R R$; however, the values of these two parameters diverge as symptom prevalence increases. We used adjusted odds ratios and reported crude (unadjusted) values of SBS symptom prevalence to estimate values of $R R$.

$$
R R=O R \frac{\left(1-P_{L}\right)_{C}}{\left(1-P_{H}\right)_{C}}
$$

where the subscript $C$ denotes the use of crude (unadjusted) symptom prevalence rates.

The data from experimental studies typically included values of ventilation rates $V_{H}$ and $V_{L}$ for direct use in equations 1, 3, 6 and 9. Cross sectional studies often provided ranges or intervals of ventilation rate associated with the values of $O R$. If suitable data were available, the average ventilation rate for buildings in each range was calculated. When such data were not available, we used the midpoint of the range as an estimate of the average.

After the original data were processed to produce values of $S_{\text {mid }}$ and $V_{\text {mid, }}$, weighted linear regressions with general estimating equations (GEE) were performed using SAS 9.1 for Windows to produce linear equations fit to the data. For the linear regression fit without GEE, a 95\% confidence band was determined using Stata 8.2. The original studies varied in sample size. If all of the studies had the same underlying "true" relationship, the slopes from each study should ideally be weighted by their precision, which is inversely proportional to variance. However, since variance information was not provided for many of the studies, regression weighted by sample size was chosen as the best alternative, because in general the higher the sample size, the lower the variance. However, study differences other than sample size, such as differences in study methods and types of symptoms, likely contributed to scatter among the slopes. To prevent the largest studies from having excessive influence on the regression, we also developed a fit to the data with the square root of sample size as the weighting factor. Analytic weights were used in both cases. 
Most studies had multiple results, e.g., from use of multiple ventilation rate categories or symptom types. Multiple results obtained from the same study have the potential to be highly correlated. The GEE routine accounts for the possible within-study correlations of outcomes. An exchangeable covariance structure was assumed. For our base case analysis, we selected the regression using GEE with weighting of studies by square root of sample size.

To derive curves of relative SBS symptom prevalence (RP) versus ventilation rate, we used the following equation, derived in the appendix of (Seppanen et al. 2006). Setting the lower limit of the integration to 10 forces RP to unity with a ventilation rate of $10 \mathrm{~L} / \mathrm{s}$-person.

$$
R P=P(V) / P(V=10)=\exp \left[\int_{10}^{V} S_{\text {mid }} d v\right]
$$

\section{RESULTS}

Table 1 provides summary information on the original studies with input data in a form suitable for use in these analyses.

Table 1. Study characteristics

\begin{tabular}{|l|c|c|c|c|}
\hline \multicolumn{1}{|c|}{ Reference } & $\begin{array}{c}\text { Study } \\
\text { Type }\end{array}$ & $\begin{array}{c}\text { No. of } \\
\text { Subjects }\end{array}$ & $\begin{array}{c}\text { SBS Symptom } \\
\text { Categories }\end{array}$ & $\begin{array}{c}\text { No. of Data } \\
\text { Points }\end{array}$ \\
\hline (Jaakkola et al. 1991) & CS & $80-484$ & Symptom Score & 9 \\
\hline (Jaakkola et al. 1994) & Ex & 75 & Al, Cn, Ey, He, Mu, Na, & 6 \\
\hline (Jaakkola and Miettinen 1995) & CS & 176,294 & Cn, Ey, He, Na & 8 \\
\hline (Mendell et al. 2005) & CS & $1160-1306$ & Lr, Mu & 12 \\
\hline (Menzies et al. 1993) & Ex & 1087 & An & 1 \\
\hline (Stenberg et al. 1994) & CC & 229,233 & SBS case & 2 \\
\hline (Tham 2004) & Ex & 56 & He & 1 \\
\hline (Wargocki et al. 2004) & Ex & 17 & Ey, Na & 2 \\
\hline
\end{tabular}

Study types: $\mathrm{CS}=$ cross sectional; Ex = experimental, $\mathrm{CC}=$ case-control

Symptom categories: $\mathrm{Al}=$ allergy, $\mathrm{An}=$ any; $\mathrm{Cn}=$ central nervous system; $\mathrm{Ey}=$ eye; $\mathrm{He}=$ headache; $\mathrm{Mu}=$ mucosal; $\mathrm{Na}=$ nasal; $\mathrm{SBS}$ case = subjects are SBS cases (with symptoms) versus controls without symptoms

Figure 1 shows the normalized slopes plotted versus midpoint ventilation rates and the fits to these data. There is considerable scatter in the data, but most slopes are negative indicating a decrease in SBS symptom prevalence with increased ventilation rate. As illustrated in the figure, the results from a technical report (Mendell et al. 2005) not reported in a journal article and the results of two studies (three data points) with SBS symptom intensity rather than prevalence as the outcome (Tham 2004; Wargocki et al. 2004) are not distinct from other results. The method used to weight the studies had only a modest impact on the resulting fit to the data. The 95\% confidence band for our base case (without GEE) excluded a slope of zero for ventilation rates between 5 and $24 \mathrm{~L} / \mathrm{s}$-person, reflecting, in part, the relative scarcity of data at high midpoint ventilation rates. The overall mean slope weighted by root sample size (not shown) is -0.027 with $95 \%$ CI $(-0.036,-0.017)$; thus, the mean slope is statistically significantly negative.

Figure 2 shows the estimated curve of relative SBS symptom prevalence (RP) plotted versus ventilation rate with RP equal to unity at a ventilation rate of $10 \mathrm{~L} / \mathrm{s}$-person, which is the minimum required ventilation rate in some codes and standards. The curve determined from GEE-based normalized slope curve is visually indistinguishable from the illustrated curve 
determined without GEE. The RP increases from 1.0 to approximately $1.23(1.12-1.32)$ [95\% confidence interval] as the ventilation rate drops from 10 to $5 \mathrm{~L} / \mathrm{s}-\mathrm{p}$. RP decreases from 1.0 to approximately $0.71(0.58-0.85)$ as ventilation rate increases from 10 to $25 \mathrm{~L} / \mathrm{s}$-person. At higher ventilation rates, the statistical uncertainty in RP becomes large relative to the estimated deviation from unity.

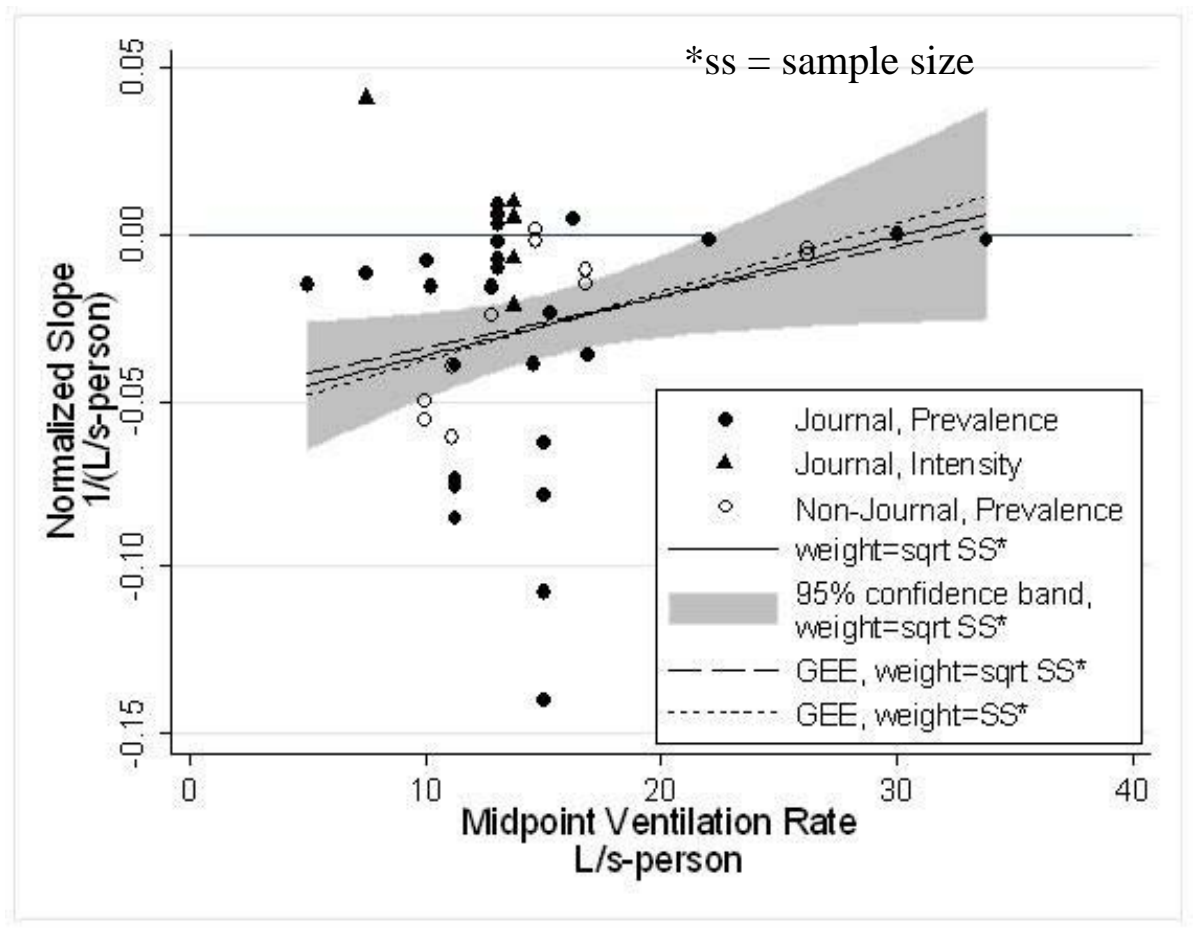

Figure 1. Normalized slopes plotted versus midpoint ventilation rates and fits to the data.

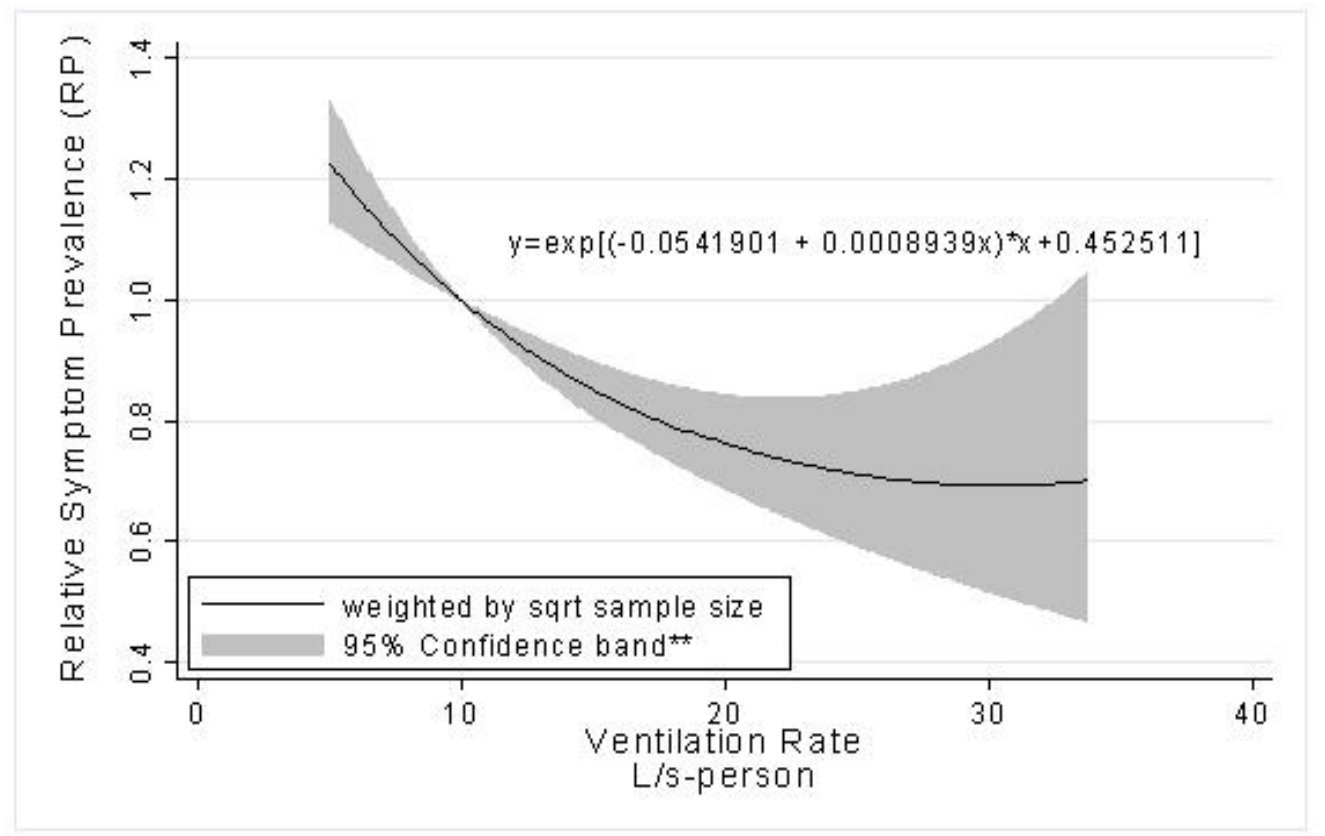

Figure 2. Estimated relative SBS symptom prevalence versus ventilation rate. ${ }^{* *}$ The $95 \%$ confidence band is based on the definite integral of the $95 \%$ confidence bands for the curve of normalized slope versus midpoint ventilation rate. 


\section{DISCUSSION}

Although prior critical reviews of the literature have concluded that lower ventilation rates are associated with higher SBS symptom prevalence rates (Seppanen et al. 1999; Wargocki et al. 2002), to the best of our knowledge this paper presents the first systematic analyses of published data to produce a quantitative estimate of how SBS symptoms vary with ventilation rates. The analyses indicate that ventilation rates have a considerable influence on symptom prevalence. For example, with a factor of two decrease in ventilation rate starting from a ventilation rate of $10 \mathrm{~L} / \mathrm{s}$-person (the minimum required value in some codes and standards), the estimated increase in symptom prevalence is approximately 23\%. Similarly, with a doubling of ventilation rate from 10 to $20 \mathrm{~L} / \mathrm{s}$-person, the estimated decrease in symptom prevalence is approximately $24 \%$.

The analyses presented here have several important limitations. The amount of original data available in a useable form was quite limited. We identified only eight useable studies, yielding only 41 data points. Because of the limited data, we did not perform distinct analyses for different types of SBS symptoms. In actuality, the relationship of ventilation rates with SBS symptoms may vary with symptom type. In addition, one would expect that the relationship of SBS symptom prevalence with ventilation rate will vary depending on the strength of indoor pollutant sources, the levels of outdoor air pollution, and other factors. Thus, the present paper provides only an estimate of the average relationship. There is no $a$ priori justification for using a linear equation, as opposed to any other type of equation, to fit the slope and ventilation-rate data. If other types of equations were fit to the data, the final curves of RP versus ventilation rate might differ significantly from those shown in figure 2 . As in most reviews and meta-analyses, our findings may also have been affected by publication bias - the tendency for studies without significant findings to less often result in publications. Publication bias would cause our predicted curve of RP versus ventilation rate to be steeper than the true curve.

Given all these limitations, it is clear that considerable uncertainty remains with respect to the quantitative relationship between SBS symptoms and ventilation rates. Despite this uncertainty, we believe that our current estimates of this relationship are much preferable to having no quantitative estimates. Consequently, we hope that these quantitative estimates will be considered a valuable input for decision making about appropriate values of minimum ventilation rates in office buildings. However, decisions about minimum ventilation rates should also consider what is known about the influence of ventilation rates on work performance (Seppanen et al. 2006), communicable respiratory disease, and perceived indoor air quality (Seppanen et al. 1999; Wargocki et al. 2002), and consider the increased energy consumption and greenhouse gas emissions associated with higher ventilation rates.

\section{CONCLUSIONS}

Systematic analyses of available data suggest that ventilation rates have a considerable influence on SBS symptom prevalence. The analyses indicate that the prevalence of SBS symptoms increases by approximately $23 \%$ as the ventilation rate drops from 10 to $5 \mathrm{~L} / \mathrm{s}-\mathrm{p}$ and decreases by approximately $29 \%$ as ventilation rate increases from 10 to $25 \mathrm{~L} / \mathrm{s}$-person. These estimates should be valuable inputs for decisions about appropriate minimum ventilation rates in office buildings. 


\section{ACKNOWLEDGEMENT}

The authors thank Phil Price and Mike Apte for their reviews of a draft of this paper and Greg Brunner at the U.S. Environmental Protection Agency for his highly effective research project management. This study was funded through interagency agreement DW89922244-01-0 between the Indoor Environments Division, Office of Radiation and Indoor Air of the U.S. Environmental Protection Agency (EPA) and the US Department of Energy under contract DE-AC02-05CH11231, to support EPA's IAQ Scientific Findings Resource Bank. Conclusions in this paper are those of the authors and not necessarily those of the U.S. EPA.

\section{REFERENCES}

Jaakkola, J. J. and P. Miettinen (1995). "Ventilation rate in office buildings and sick building syndrome." Occup Environ Med 52(11): 709-14.

Jaakkola, J. J., P. Tuomaala and O. Seppanen (1994). "Air recirculation and sick building syndrome: a blinded crossover trial." Am J Public Health 84(3): 422-8.

Jaakkola, J. K. K., L. M. Reinikainen, O. P. Heinonen, A. Majanen and O. Seppanen (1991). "Indoor air requirements for healthy office buildings: recommendations based on an epidemiologic study." Environment International 17: 371-378.

Mendell, M. J., Q. H. Lei, M. G. Apte and W. J. Fisk (2005). Outdoor air ventilation and work-related symptoms in U.S. office buildings - results from the BASE study. LBNL-56381. Berkeley, CA, Lawrence Berkeley National Laboratory.

Menzies, R., R. Tamblyn, J. P. Farant, J. Hanley, F. Nunes and R. Tamblyn (1993). "The effect of varying levels of outdoor-air supply on the symptoms of sick building syndrome." N Engl J Med 328(12): 821-7.

Seppanen, O., W. J. Fisk and Q. H. Lei (2006). "Ventilation and performance in office work." Indoor Air 16(1): 28-36.

Seppanen, O. A., W. J. Fisk and M. J. Mendell (1999). "Association of ventilation rates and $\mathrm{CO}_{2}$ concentrations with health and other responses in commercial and institutional buildings." Indoor Air 9(4): 226-52.

Stenberg, B., N. Eriksson, J. Hoog, J. Sundell and S. Wall (1994). "The sick building syndrome (SBS) in office workers. A case referent study of personal, psychosocial and building-related risk indicators." International Journal of Epidemiology 23: 11901197.

Tham, K. W. (2004). "Effects of temperature and outdoor air supply rate on the performance of call center operators in the tropics." Indoor Air 14 Suppl 7: 119-25.

Wargocki, P., J. Sundell, W. Bischof, G. Brundrett, P. O. Fanger, F. Gyntelberg, S. O. Hanssen, P. Harrison, A. Pickering, O. Seppanen and P. Wouters (2002). "Ventilation and health in non-industrial indoor environments: report from a European multidisciplinary scientific consensus meeting (EUROVEN)." Indoor Air 12(2): 11328.

Wargocki, P., D. P. Wyon and P. O. Fanger (2004). "The performance and subjective responses of call-center operators with new and used supply air filters at two outdoor air supply rates." Indoor Air 14 Suppl 8: 7-16. 